\title{
Rat bone mesenchymal stem cells exert antiproliferative effects on nicotine-exposed $T$ cells via iNOS production
}

\author{
XIAOYAN LI ${ }^{1}$, JIANYING XU ${ }^{1}$ and PINGPING $\mathrm{LI}^{2}$ \\ ${ }^{1}$ Department of Respiratory and Critical Care Medicine, Shanxi Academy of Medical Sciences, \\ Shanxi Bethune Hospital, Taiyuan, Shanxi 030032; ${ }^{2}$ Department of Respiratory and Critical Care Medicine, \\ The Second Clinical Medical College, Shanxi Medical University, Taiyuan, Shanxi 030009, P.R. China
}

Received June 20, 2019; Accepted February 14, 2020

DOI: $10.3892 / \mathrm{mmr} .2020 .11027$

\begin{abstract}
Adoptive transfer of bone marrow-derived mesenchymal stem cells (BMSCs) significantly alleviates smoking-induced chronic obstructive pulmonary disease (COPD) in rats. Considering the critical roles of $\mathrm{T}$ cells during COPD development, the present study aimed to further identify the molecular mechanisms underlying the antiproliferative effect of BMSCs on splenic $\mathrm{T}$ cells isolated from rats following chronic exposure to nicotine. Splenic T cells were co-cultured with rat BMSCs at various ratios and subsequently, T-cell proliferation was measured using the Cell Counting Kit- 8 assay. The effects of the inducible nitric oxide synthase (iNOS) inhibitor N-nitro-L-arginine methylester (L-NAME) and short hairpin (sh)RNA-lentivirus-mediated knockdown of iNOS in BMSCs on T-cell proliferation were evaluated. The expression levels of iNOS and STAT5 phosphorylation in BMSCs and T cells, respectively, were assessed by reverse transcription-quantitative PCR and western blotting. A higher ratio of BMSCs to $\mathrm{T}$ cells resulted in increased inhibition of T-cell proliferation; therefore, the ratio of 1:20 was selected for further in vitro experiments. At a dose of $5 \mu \mathrm{M}$, L-NAME displayed the strongest ability to reverse the antiproliferative effects of BMSCs in the co-culture system. Both L-NAME treatment and shRNA-mediated knockdown of iNOS expression significantly decreased the suppressive effects of BMSCs, downregulated iNOS expression at the mRNA and protein levels in BMSCs, and enhanced STAT5 phosphorylation in $\mathrm{T}$ cells. BMSCs inhibited the proliferation of nicotine-exposed $\mathrm{T}$ cells, which was associated with iNOS expression in BMSCs
\end{abstract}

Correspondence to: Dr Xiaoyan Li, Department of Respiratory and Critical Care Medicine, Shanxi Academy of Medical Sciences, Shanxi Bethune Hospital, 99 Longcheng Avenue, Taiyuan, Shanxi 030032, P.R. China

E-mail: xy740922@163.com

Key words: bone marrow-derived mesenchymal stem cells, chronic obstructive pulmonary disease, chronic cigarette smoke exposure, T-cell proliferation, inducible nitric oxide synthase, signal transducer and activator of transcription and decreased STAT5 phosphorylation in T cells. The present study indicated the potential mechanisms underlying the beneficial effects of BMSC infusion in patients with chronic smoking-induced COPD and emphysema.

\section{Introduction}

Patients with chronic obstructive pulmonary disease (COPD) suffer from persistent respiratory symptoms and limited airflow. As an obstructive disease of the lungs, COPD affects $>150$ million individuals worldwide and caused 3.2 million deaths in 2015 globally $(1,2)$. Tobacco smoking-induced lung inflammation and damage are the most common causes of COPD $(3,4)$; however, the exact mechanisms underlying long-term cigarette smoking-induced immune dysregulation are not completely understood $(5,6)$. It has been reported that the accumulation of activated innate immune cells, including macrophages and adaptive immune responses contribute to the pathophysiology of COPD (7).

In mouse models of cigarette smoke exposure-induced COPD, emphysematous alterations in the lungs, which are accompanied by altered lung function and infiltration of inflammatory cells and $\mathrm{T}$ cells, have been identified $(8,9)$. Clinical studies have reported that oligoclonal $\mathrm{T}$ cells accumulate in the airway wall and lungs of patients with COPD, and the degree of T-lymphocyte infiltration positively correlates with the severity of airflow limitation and emphysema $(10,11)$. Animal studies have also demonstrated that cigarette smoking induces the accumulation of lymphocytes in the lungs of mice with emphysema, whereas CD4 ${ }^{+}$ and $\mathrm{CD} 8^{+} \mathrm{T}$ cell knockout mice do not display emphysema after long-term exposure to cigarette smoke $(12,13)$. The aforementioned results suggest that $\mathrm{T}$ cell-mediated adaptive immunity plays an important role during the development of COPD and emphysema.

Mesenchymal stem cells (MSCs) are multipotent non-hematopoietic cells that exert immunosuppressive effects on a number of different immune cells, including dendritic cells (14), B lymphocytes (15) and T lymphocytes (16-20). MSCs can secrete a large number of chemokines and immunosuppressive factors when stimulated by inflammatory factors (21). As a result, lymphocytes can be recruited by chemotaxis to the location where the MSCs reside and subsequently 
inhibited by the high local concentration of immunosuppressive factors (22). In particular, it has been reported that bone marrow-derived MSCs (BMSCs) significantly decrease T-lymphocyte proliferation by secreting transforming growth factor (TGF)- $\beta 1$, hepatocyte growth factor (HGF) (16), prostaglandins (19), nitric oxide (NO) (20) and inducible nitric oxide synthase (iNOS) (23), an enzyme that catalyzes the production of NO. Furthermore, the antiproliferative effect of rat BMSCs on $\mathrm{T}$ lymphocytes has been demonstrated to be mediated by the induced expression of iNOS and production of NO by BMSCs (23). A previous study also suggested that tail vein injection of BMSCs in rats significantly reduces cigarette smoking-induced downregulation of iNOS expression in the blood circulation and lungs of rats (24). In addition, adoptive transfer of BMSCs alleviates lung inflammation and injury, and was associated with reduced STAT5 phosphorylation in lung-infiltrating $\mathrm{T}$ lymphocytes (24). However, although it has been reported that iNOS regulates $\mathrm{T}$ cell death and immune memory (25), and rat BMSCs exert immunoregulatory effects in an animal model of COPD, whether BMSCs directly inhibit the proliferation of nicotine-exposed T cells via iNOS expression and inhibition of STAT5 phosphorylation in T cells is not completely understood.

In the present study, splenic $\mathrm{T}$ cells were isolated from rats after chronic nicotine exposure to establish an in vitro BMSC:T cell co-culture system. Following treatment of the co-cultured cells with an iNOS inhibitor or lentivirus infection-mediated silencing of iNOS in rat BMSCs, the contributions of iNOS and STAT5 phosphorylation to the antiproliferative effect of BMSCs on nicotine-exposed $\mathrm{T}$ cells were investigated.

\section{Materials and methods}

Animal model of nicotine exposure. A total of 30 male Sprague-Dawley rats (age, 8-10 weeks; weight, $\sim 120 \mathrm{~g}$ ) were purchased from the Charles River Laboratories and housed in the specific pathogen-free facility at the Experimental Animal Center of Shanxi Medical University. Animals were maintained at room temperature $\left(22 \pm 1^{\circ} \mathrm{C}\right)$, in $60-70 \%$ humidity, with 12-h light/dark cycles and access to food and water ad libitum. Nicotine exposure to rats was administered via cigarette smoke, as previously reported (24). Briefly, rats were placed in an organic glass passive smoking cage and exposed to the cigarette smoke of 20 filtered commercial cigarettes for $1 \mathrm{~h}$, twice per day for 6 days per week, for a total of 24 weeks. Under these conditions, individual rats were exposed to $11 \mathrm{mg}$ tar and $0.9 \mathrm{mg}$ nicotine during each $1 \mathrm{~h}$ exposure. All animal experiments in the present study were approved by the Animal Care and Research Committee of Shanxi Medical University and performed with strict adherence to the Guide for the Care and Use of Laboratory Animals (8th edition) (26).

BMSC isolation, culture and characterization. Male Sprague-Dawley rats (age, 4-6 weeks; weight, $\sim 100 \mathrm{~g} ; \mathrm{n}=6$; purchased from Charles River Laboratories and housed as previously described above) were euthanized with $\mathrm{CO}_{2}$ with $10-30 \%$ volume displacement rate $/ \mathrm{min}$. Bone marrow cells were isolated from the femurs and tibias of the rats by flushing the bone marrow cavity with complete DMEM (Gibco; Thermo Fisher Scientific, Inc.), supplemented with 10\% FBS
(Gibco; Thermo Fisher Scientific, Inc.). Cells were inoculated into a cell culture flask and cultured with $5 \% \mathrm{CO}_{2}$ at $37^{\circ} \mathrm{C}$ for 3 days. Subsequently, the unattached cells were removed and the remaining cells were incubated for 9-10 days. At 80-90\% confluency, BMSC-enriched cells displayed a typical long fusiform shape and were passaged at a ratio of 1:2 or 1:3. BMSCs at passage 3 were used for subsequent experiments. Flow cytometric staining of surface markers $\left(\mathrm{CD} 34^{+} \mathrm{CD} 45^{-} \mathrm{CD} 90^{-}\right)$was performed to evaluate the purity of the BMSCs, as previously described (27). BMSCs with $>90 \%$ purity were used for the co-culture with nicotine-exposed rat $\mathrm{T}$ cells.

T-cell isolation and culture. The spleens were also aseptically isolated from the Sprague-Dawley rats (age, 32-34 weeks) that had been chronically exposed to nicotine for 24 weeks and euthanized as described above. Spleen tissues were repeatedly cut into small pieces with ophthalmic scissors to generate a splenocyte homogenate in RPMI-1640 medium (Wuhan Boster Biological Technology, Ltd.) supplemented with 10\% FBS. The homogenate was passed through a $100-\mu \mathrm{m}$ cell strainer and the subsequent cell suspension was subjected to density gradient centrifugation at $20^{\circ} \mathrm{C}$ and $400 \mathrm{x}$ g for 20 min with a lymphocyte separation solution (TBD Sciences), according to the manufacturer's protocol. Cells in the lymphocyte layer were transferred to a separate tube and washed twice with complete RPMI- 1640 medium at $400 \mathrm{x}$ g and $20^{\circ} \mathrm{C}$ for $5 \mathrm{~min}$. Cells were inoculated into $75-\mathrm{cm}^{2}$ cell culture flasks at a cell density of $1.0 \times 10^{6} / \mathrm{ml}$ and T-cell stimulating agent ConA $(5 \mu \mathrm{g} / \mathrm{ml}$; Sigma-Aldrich; Merck KGaA) and T-cell growth factor interleukin-2 (20 ng/ml; PeproTech, Inc.) were added to the medium. Following incubation for 3 days, the T cells began growing and displayed a round or elliptical shape, which was similar to what has been previously described of activated cells (28).

BMSC and T cell co-culture. BMSCs in the logarithmic growth phase were seeded into 96 -well plates $(100 \mu \mathrm{l} /$ well $)$ at the following densities: $1 \times 10^{3}, 2 \times 10^{3}, 4 \times 10^{3}, 8 \times 10^{3}$ and $16 \times 10^{3}$ cells/well. BMSCs were incubated overnight to allow cell attachment. Subsequently, T lymphocytes isolated from the spleens of nicotine-exposed rats were added to the wells $\left(3.2 \times 10^{5}\right.$ cells/well). The corresponding ratios of BMSCs to $\mathrm{T}$ lymphocytes were: $1: 320,1: 160,1: 80,1: 40$ and 1:20, respectively. The total volume of the BMSC/T cell co-culture system in each well of the 96-well plate was $200 \mu$ l. After co-culture for $48 \mathrm{~h}$, the suspended T lymphocytes were transferred to another 96-well plate. Cells were observed using an inverted phase contrast light microscope (magnification, $\mathrm{x} 4$ or $\mathrm{x} 10)$ and cell growth was measured. In certain cases, different concentrations $(0.5,1,5,10$ or $20 \mu \mathrm{M})$ of the iNOS inhibitor N-nitro-L-arginine methylester (L-NAME; Selleck Chemicals) were added to the co-culture at the same time as the T cells. In other cases, lentivirus-infected (control or iNOS knockdown-targeting) BMSCs were used for the co-culture with $\mathrm{T}$ cells.

Cell proliferation evaluation using the cell counting kit-8 (CCK-8) assay. The antiproliferative effect of BMSCs on $\mathrm{T}$ cells was determined using the CCK- 8 assay (Dojindo Molecular Technologies, Inc.), according to the manufacturer's 
protocol. Briefly, after BMSCs and T cells were co-cultured for $48 \mathrm{~h}$, T cells were transferred to a new plate, $20 \mu \mathrm{l} \mathrm{CCK}-8$ reagent was added to each well and cells were incubated for $4 \mathrm{~h}$ at $37^{\circ} \mathrm{C}$. The optical density value of each well was determined at a wavelength of $450 \mathrm{~nm}$ with a reference wavelength of $600 \mathrm{~nm}$ using a microplate reader. The assay was repeated three times and the samples were assayed in triplicate.

Knockdown of iNOS in BMSCs using a small hairpin (sh)RNA lentivirus. The shRNA sequence (5'-CCACTAACA GTGGCAACAT-3') and a random negative control sequence (5'-CGAGGGCGACTTAACCTTAGG-3') were cloned into the pLV(shRNA)-EGFP/Puro-U6 lentiviral vector (Cyagen Biosciences, Inc.). The iNOS-specific shRNA sequence was located at the coding sequence locus of the NOS2 gene (GenBank accession no.NM_012611.3).293T cells (American Type Culture Collection) were plated onto $10-\mathrm{cm}$ dishes at a density of $6 \times 10^{6}$ cells/dish and were co-transfected with control lentiviral vector (CV; $10 \mu \mathrm{g} / \mathrm{dish})$ or iNOS-knockdown lentiviral vector (iNOS-shRNA; $10 \mu \mathrm{g} / \mathrm{dish}$ ) together with helper plasmids pMD2.G (Addgene, Inc.; $5 \mu \mathrm{g} / \mathrm{dish}$ ) and psPAX2 (Addgene, Inc.; $5 \mu \mathrm{g} / \mathrm{dish}$ ) using Lipofectamine $2000^{\circledR}$ transfection reagent (Thermo Fisher Scientific, Inc.), according to the manufacturer's protocol. Vector construction, verification by sequencing, virus packaging and collection of the corresponding viral supernatants following $48 \mathrm{~h}$ of transfection were performed by Cyagen Biosciences, Inc. Subsequently, $2.5 \times 10^{5} \mathrm{BMSC} /$ well at $70 \%$ confluence were seeded into 6 -well plates and were infected with the lentiviral supernatant at a multiplicity of infection of 30 . Following $48 \mathrm{~h}$ of infection, BMSCs were used for subsequent experiments.

Western blotting. BMSCs and T cells were harvested and sonicated at the frequency of $20 \mathrm{kHz}$ for $10 \mathrm{sec}$ at $4^{\circ} \mathrm{C}$ in phenylmethanesulfonylfluoride-containing RIPA lysis buffer (Thermo Fisher Scientific, Inc.) and then incubated with RIPA lysis buffer for $30 \mathrm{~min}$ on ice. Subsequently, cell lysates were centrifuged at $10,000 \mathrm{x}$ g for $15 \mathrm{~min}$ at $4^{\circ} \mathrm{C}$. Total protein in the supernatant was quantified using the bicinchoninic acid reagent kit (Thermo Fisher Scientific, Inc.), according to the manufacturer's protocol. Proteins $(30-50 \mu \mathrm{g})$ were separated by $10 \%$ SDS-PAGE and transferred onto nitrocellulose membranes. Subsequently, membranes were blocked with $5 \%$ skim milk for $2 \mathrm{~h}$ at room temperature and incubated overnight at $4{ }^{\circ} \mathrm{C}$ with primary antibodies targeted against: iNOS (1:250; cat. no. ab49999; Abcam), STAT5 (1:500; cat. no. ab230670; Abcam), phosphorylated STAT5 (1:1,000; cat. no. 05-495; EMD Millipore) and $\beta$-actin (1:500; cat. no. MA1115; Wuhan Boster Biological Technology, Ltd.). Following the primary incubation, membranes were washed with TBS containing $0.5 \%$ Tween 20 and incubated for $2 \mathrm{~h}$ at room temperature with a horseradish peroxidase-conjugated goat anti-mouse IgG secondary antibody (1:10,000; cat. no. TA130003; OriGene Technologies, Inc.). Protein bands were visualized using an enhanced chemiluminescence kit (EMD Millipore). Blots were performed in triplicate and protein expression was quantified using AlphaView version 3.4 software (ProteinSimple) with $\beta$-actin as the loading control.
Reverse transcription-quantitative PCR (RT-qPCR). Total RNA was extracted from BMSCs using TRIzol ${ }^{\circledR}$ reagent (Invitrogen; Thermo Fisher Scientific, Inc.), according to the manufacturer's protocol. RNA quality was confirmed by $1 \%$ agarose gel electrophoresis. Total RNA $(1 \mu \mathrm{g})$ was reverse transcribed into cDNA at $37^{\circ} \mathrm{C}$ for 15 min using the PrimeScript ${ }^{\circledR}$ RT Master Mix Perfect Real Time Reagent kit (Takara Bio, Inc.), according to the manufacturer's protocol. Subsequently, qPCR was performed using FastStart Universal SYBR Green Master (ROX; Sigma-Aldrich; Merck KGaA) and an AB7500 RT-PCR instrument (Applied Biosystems; Thermo Fisher Scientific, Inc.). The following primer pairs were used for qPCR: iNOS forward, 5'-CACCTTGGAGTT CACCCAGT-3' and reverse, 5'-ACCACTCGTACTTGGGAT GC-3'; $\beta$-actin forward, 5'-GTCAGGTCATCACTATCG GCAAT-3' and reverse, 5'-AGAGGTCTTTACGGATGTCAA CGT-3'. The following thermocycling conditions were used for qPCR: Initial denaturation for $10 \mathrm{~min}$ at $95^{\circ} \mathrm{C} ; 40$ cycles of denaturation for $5 \mathrm{sec}$ at $95^{\circ} \mathrm{C}$ and annealing and extension for $1 \mathrm{~min}$ at $60^{\circ} \mathrm{C}$; followed by a melting curve analysis. RT-qPCR was repeated three times and each sample was tested in triplicate. mRNA expression levels were quantified using the $2^{-\Delta \Delta \mathrm{Cq}}$ method (29) and normalized to the internal reference gene $\beta$-actin.

Statistical analysis. Statistical analyses were performed using SPSS software (version 13.0; SPSS, Inc.). Data are expressed as the mean \pm standard deviation of $\geq 3$ experimental repeats. Multiple comparisons were performed using one-way ANOVA followed by Tukey's post hoc test. $\mathrm{P}<0.05$ was considered to indicate a statistically significant difference.

\section{Results}

Co-culture of higher ratios of BMSCs:T cells results in increased suppression of T-cell proliferation. The present study aimed to establish a BMSC:T cell co-culture system for evaluating the antiproliferative effect of BMSCs on T cells in vitro. To determine the most suitable ratio of BMSCs:T cells that resulted in the most potent inhibition of T-cell proliferation, rat BMSCs were co-cultured with rat splenocyte-derived $\mathrm{T}$ cells at various ratios. An increasing number of BMSCs $\left(1 \times 10^{3}-16 \times 10^{3}\right.$ cells/well) were seeded into 96 -well plates and co-cultured with a fixed number of $\mathrm{T}$ cells isolated from nicotine-exposed rats $\left(3.2 \times 10^{5}\right.$ cells/well $)$. An increase in the number of BMSCs increased the inhibition of T-cell proliferation, as indicated by the CCK-8 assay (Fig. 1A). At a BMSC:T cell ratio of $1: 20$, maximal proliferation inhibition was observed ( $>90 \%$ compared with the control group; Fig. 1A). The results suggested that a higher ratio of BMSCs:T cells resulted in increased inhibition of T-cell proliferation. Due to the limited capacity of the 96-well plate, 1:20 was the highest ratio that could be investigated in the present study; therefore, the ratio of 1:20 BMSCs:T cells was selected for subsequent experiments.

iNOS inhibitor L-NAME at $5 \mu M$ displays the maximal ability to reverse the antiproliferative effects of BMSCs. To investigate the effect of iNOS on the suppressive function of BMSCs, the culture medium of the BMSC:T-cell co-culture system was 

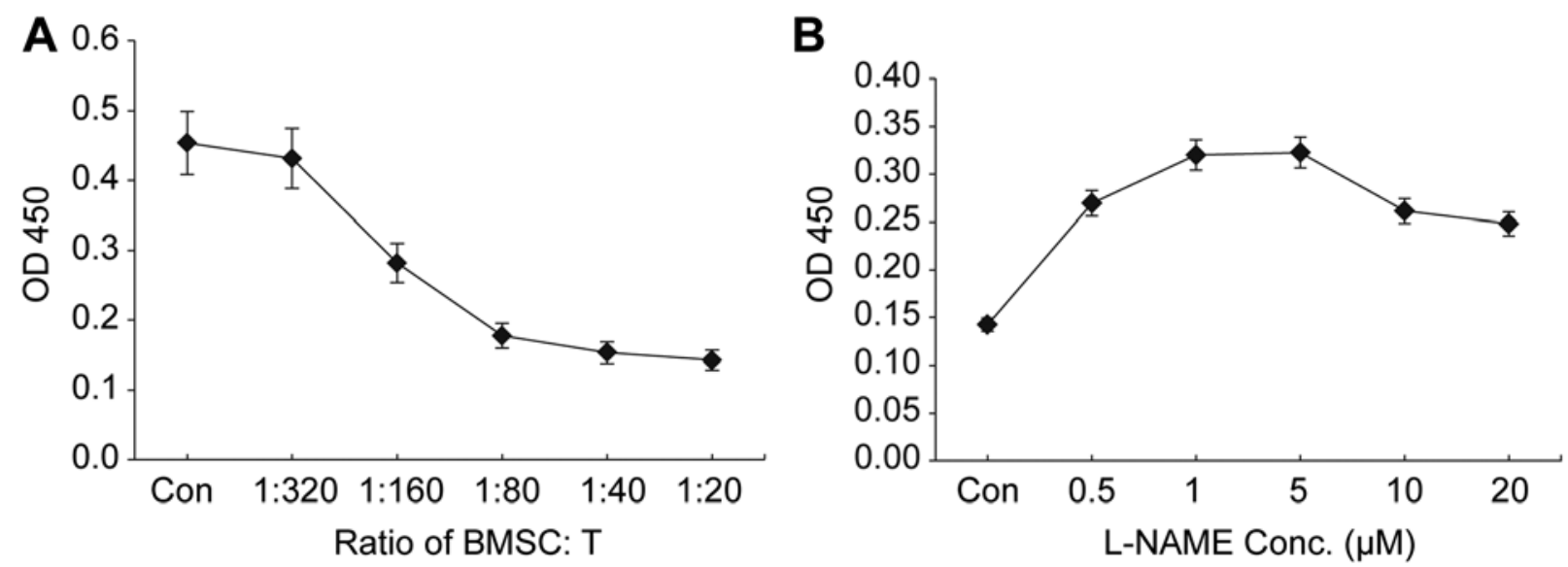

Figure 1. Effects of different ratios of BMSCs:T cells and different concentrations of the iNOS inhibitor L-NAME on the in vitro BMSC:T cell co-culture system. (A) The proliferation of T cells co-cultured with BMSCs at different ratios was measured using the CCK-8 assay. (B) The proliferation of T cells co-cultured with BMSCs at a ratio of 1:20 and treated with different concentrations of L-NAME was measured using the CCK-8 assay. BMSCs, bone marrow-derived mesenchymal stem cells; iNOS, inducible nitric oxide synthase; L-NAME, N-nitro-L-arginine methylester; CCK-8, Cell Counting Kit-8; OD, optical density; Con, control; conc, concentration.

supplemented with the iNOS inhibitor L-NAME at various concentrations. When BMSCs and T cells were co-cultured at the ratio of 1:20, addition of L-NAME (starting from $0.5 \mu \mathrm{M}$ ) reversed BMSC-mediated inhibition of T-lymphocyte proliferation in a concentration-dependent manner with L-NAME concentrations $\leq 5 \mu \mathrm{M}$, and L-NAME at $5 \mu \mathrm{M}$ displayed the maximal reversal effect (Fig. 1B). Compared with L-NAME at $5 \mu \mathrm{M}$, higher concentrations of L-NAME $(10$ and $20 \mu \mathrm{M})$ resulted in decreased proliferation of co-cultured $\mathrm{T}$ cells; therefore, L-NAME at a dose of $5 \mu \mathrm{M}$ was used for further investigation of iNOS-mediated mechanisms underlying the antiproliferative effects of BMSCs on T cells.

Functional inactivation of iNOS by inhibitor treatment or shRNA-mediated knockdown reverses the suppressive effects of BMSCs. To further investigate the role of iNOS in mediating the antiproliferative effect of BMSCs on T cells, an iNOS shRNA-expressing lentivirus was generated. BMSCs were infected with either CV or shRNA-iNOS. Following incubation for $48 \mathrm{~h}$, transfected cells were co-cultured with $\mathrm{T}$ lymphocytes isolated from nicotine-exposed rats at the ratio of 1:20. BMSCs in the control and CV groups significantly inhibited the proliferation of T lymphocytes. By contrast, treatment with the iNOS inhibitor L-NAME or silencing of iNOS expression significantly reversed the antiproliferative effects of BMSCs (Fig. 2).

Antiproliferative effect of BMSCs is dependent on iNOS production by BMSCs and is associated with a reduction in STAT5 phosphorylation in T cells. A previous study on the transfusion of BMSCs into nicotine-exposed rats indicated that the suppressive roles of BMSCs in autoimmune responses were associated with reductions in serum and lung iNOS expression levels and smoking-induced STAT5 phosphorylation in lung tissue-derived lymphocytes (24). To investigate whether STAT5 phosphorylation was also associated with the antiproliferative effects of BMSCs in the direct BMSC: T-cell co-culture system, the expression levels of iNOS in BMSCs and STAT5 in T cells were assessed. As determined by

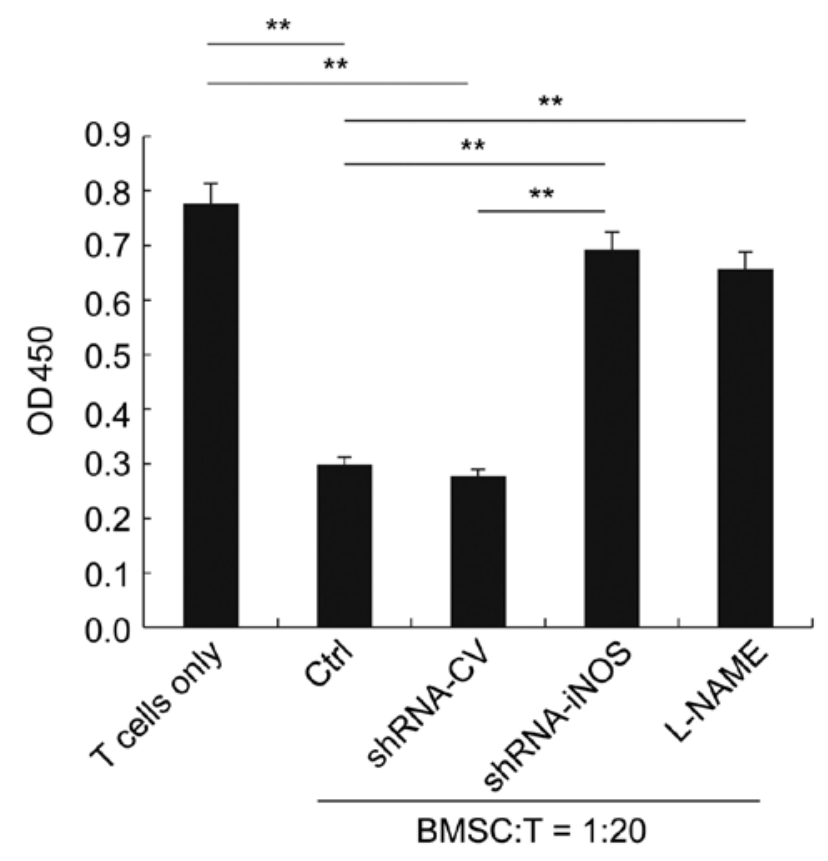

Figure 2. iNOS inhibitor treatment and shRNA-mediated knockdown of iNOS significantly reverse the antiproliferative effect of BMSCs on $\mathrm{T}$ cells. T cells were co-cultured with control, shRNA-CV-transduced, shRNA-iNOS-transduced or L-NAME-treated rat BMSCs at a ratio of 1:20. $\mathrm{T}$ cells cultured alone were considered as the negative control. ${ }^{* * *} \mathrm{P}<0.01$, as indicated. iNOS, inducible nitric oxide synthase; shRNA, small hairpin RNA; BMSCs, bone marrow-derived mesenchymal stem cells; CV, control lentivirus; L-NAME, N-nitro-L-arginine methylester; OD, optical density; Ctrl, control.

RT-qPCR, compared with the corresponding control groups, the L-NAME and shRNA-iNOS groups displayed significantly downregulated iNOS expression levels in BMSCs (Fig. 3A). Consistently, the western blotting results also indicated that the protein expression levels of iNOS were significantly reduced in the L-NAME and shRNA-iNOS groups compared with the corresponding control groups (Fig. 3B and C). Furthermore, the level of STAT5 phosphorylation was significantly 


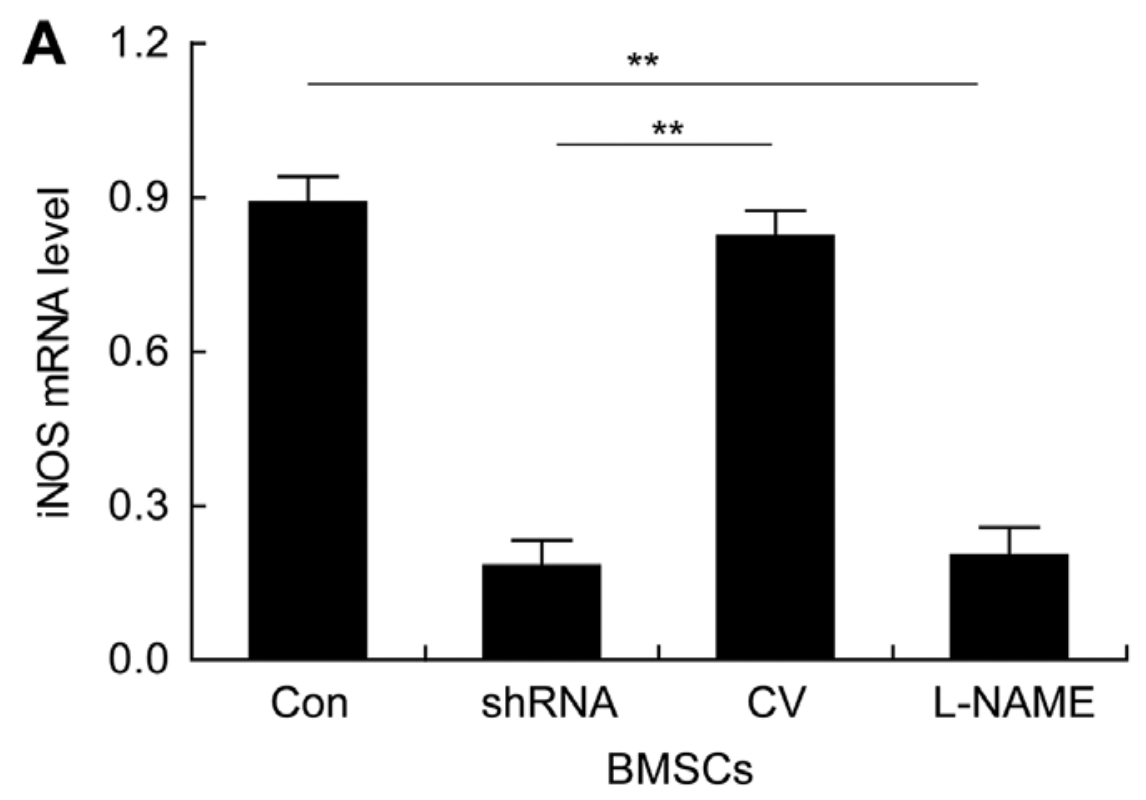

B
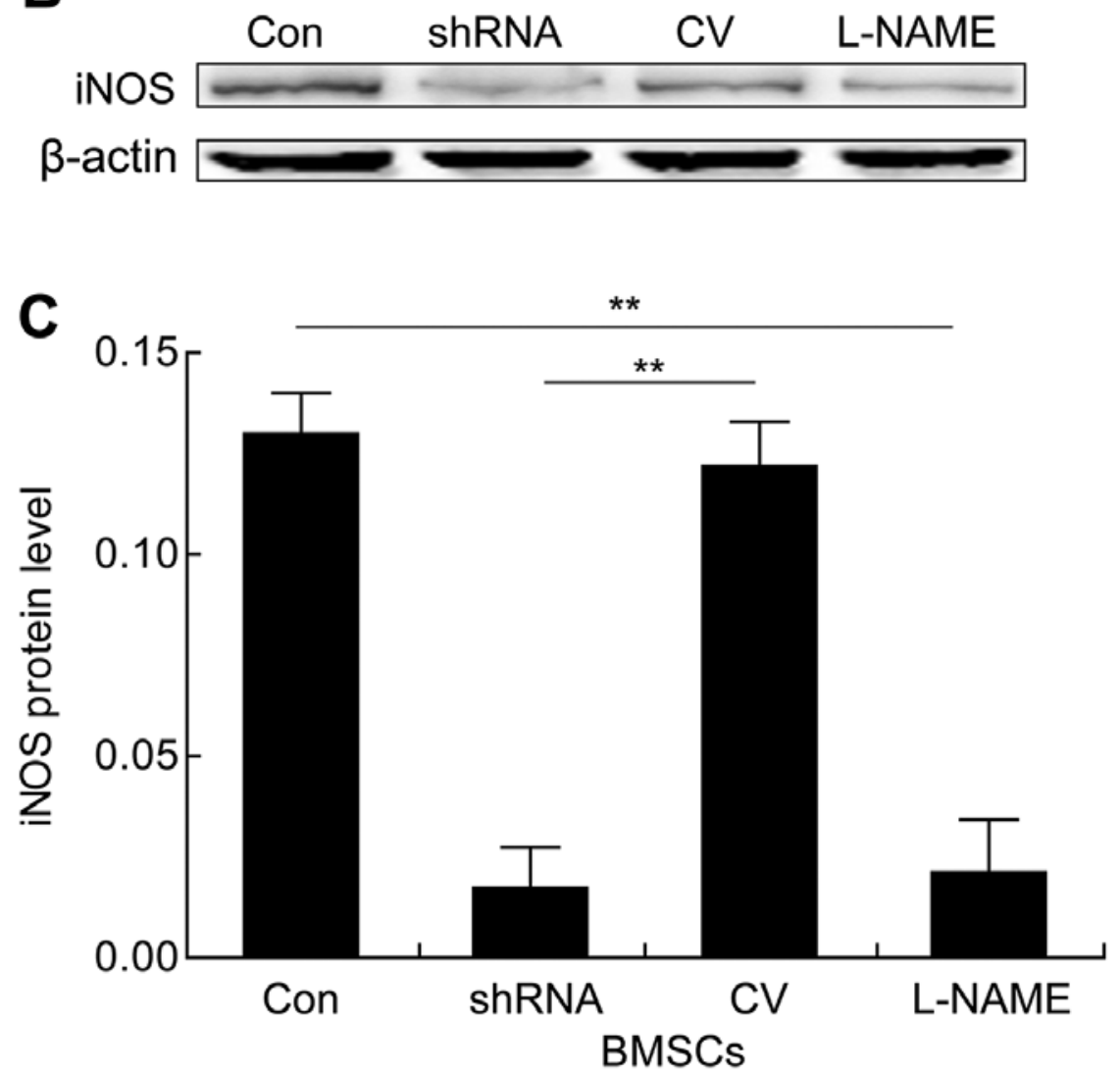

Figure 3. iNOS inhibitor treatment and shRNA-mediated knockdown of iNOS significantly reduce the expression levels of iNOS in BMSCs. (A) iNOS mRNA expression levels were determined by reverse transcription-quantitative PCR. iNOS protein expression levels were (B) determined by western blotting and (C) quantified. ${ }^{* *} \mathrm{P}<0.01$, as indicated. iNOS, inducible nitric oxide synthase; shRNA, short hairpin RNA; BMSCs, bone marrow-derived mesenchymal stem cells; Con, control; CV, control lentivirus; L-NAME, N-nitro-L-arginine methylester.

increased in T cells co-cultured with BMSCs with reduced iNOS expression compared with the corresponding control groups (Fig. 4A). The increased expression of phosphorylated STAT5 resulted in significantly increased ratios of phosphorylated STAT5/total STAT5 expression in the L-NAME and shRNA-iNOS groups compared with the corresponding control groups (Fig. 4B). Collectively, the results suggested that iNOS expression in BMSCs was negatively associated with STAT5 phosphorylation in T cells in the co-culture system. Furthermore, the results indicated that iNOS production in BMSCs and reduced STAT5 phosphorylation in T cells contributed to the antiproliferative effects of BMSCs. 
A

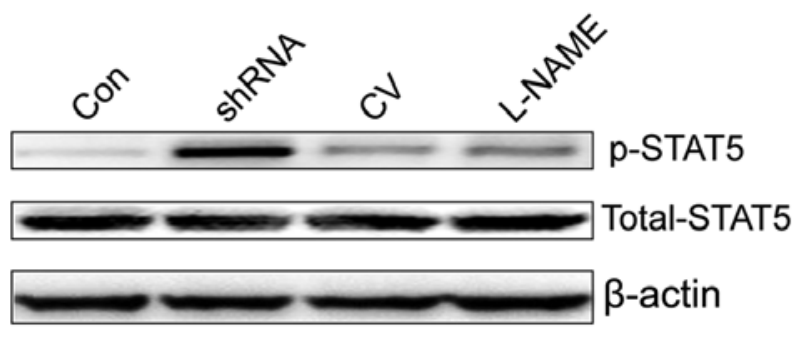

B

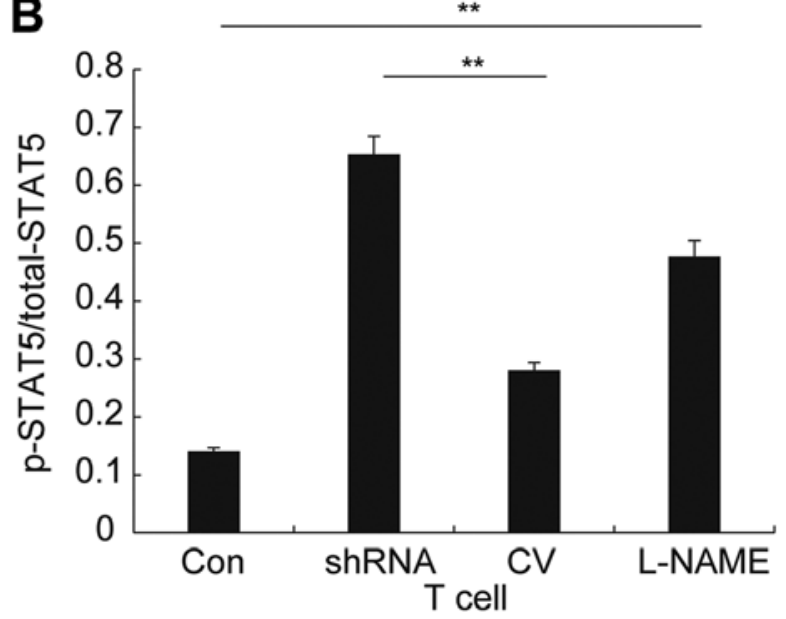

Figure 4. iNOS inhibitor treatment and shRNA-mediated knockdown of iNOS in BMSCs significantly increases STAT5 phosphorylation in co-cultured $\mathrm{T}$ cells. The protein expression levels of phosphorylated STAT5 and total STAT5 were (A) determined by western blotting and (B) quantified. ${ }^{* *}$ P $<0.01$, as indicated. iNOS, inducible nitric oxide synthase; shRNA, short hairpin RNA; BMSCs, bone marrow-derived mesenchymal stem cells; p, phosphorylated; Con, control; CV, control lentivirus; L-NAME, N-nitro-L-arginine methylester.

\section{Discussion}

In previous years, the role of T cell-mediated immune regulation in the pathogenesis of COPD has gained increasing attention. Long-term cigarette smoking is a risk factor for COPD and lung-infiltrating $\mathrm{T}$ lymphocytes play an indispensable role during the development of COPD and emphysema $(30,31)$. MSCs with strong immunosuppressive properties have been reported to attenuate COPD and emphysema progression in animal models and human clinical trials (32-34). In the present study, the suppressive effect of rat BMSCs on the proliferation of splenic $\mathrm{T}$ cells isolated from chronic nicotine-exposed rats was investigated in an in vitro BMSC and $\mathrm{T}$ cell co-culture system. The results indicated that the suppressive function of BMSCs on T-cell proliferation was dependent on the expression of iNOS in BMSCs and reduced STAT5 phosphorylation in T cells.

A previous study reported that chronic cigarette smoke exposure induces immune dysregulation in the lungs of rats and adoptive transfer of BMSCs significantly attenuates the imbalance of proinflammatory and anti-inflammatory factors, reduces the antibody response to lung elastin antigen, and attenuates chronic inflammatory damage in the lungs (24). Furthermore, analysis of the cell cycle demonstrated that T-lymphocyte proliferation in the lungs of rats treated with BMSC adoptive transfer following exposure to cigarette smoke is arrested at the $G_{0} / G_{1}$ phase, which correlates with the reduced level of inflammatory mediators and local inflammatory responses (24). However, the molecular mechanisms underlying MSC-mediated inhibition of T-lymphocyte proliferation and immune response regulation are not completely understood $(16,17,35-38)$. The hypothesis that a soluble molecule mediates the inhibitory effect of MSCs on T cells remains controversial. Certain previous studies have reported that TGF- $\beta$, HGF, indoleamine 2,3-dioxygenase (IDO) and prostaglandin E2 mediate the suppressive effects of MSCs on T cells $(16,38,39)$. By contrast, other previous studies have reported that MSCs inhibit T lymphocytes via contact-depen- dent mechanisms $(18,40)$ and cellular stress induction (41). Previous studies conducted by Su et al (42) and Shi et al (43) revealed that the key molecule mediating immunosuppression by MSCs is species dependent; MSCs from monkeys, pigs and humans suppress immune responses via IDO, whereas MSCs from mice, rats, rabbits and hamsters mediate immune responses via iNOS. MSCs derived from iNOS knockout mice displayed significantly reduced antiproliferative effects on T lymphocytes (20). In addition, it has been indicated that the antiproliferative effect of rat BMSCs on T lymphocytes is mediated by the induction of iNOS expression and production of NO by BMSCs (23). Therefore, the present study aimed to determine whether BMSCs directly inhibited the proliferation of nicotine-exposed T cells via iNOS expression, while the effects of other soluble factors, including TGF- $\beta$ and prostaglandins, require further investigation. Consistently, a previous study also demonstrated that tail vein injection of BMSCs significantly reduced the downregulation of iNOS levels in the blood circulation and lungs of rats chronically exposed to cigarette smoke (24). The critical role of iNOS in mediating the suppressive function of BMSCs was further suggested by the results of the direct cell co-culture experiments performed in the present study. Functional inactivation of iNOS by shRNA-mediated iNOS silencing or treatment with an iNOS inhibitor significantly reversed the suppressive function of BMSCs during co-culture with T cells. In the present study, BMSCs at passage 3 were used and the co-culture period was relatively short ( 24 or $48 \mathrm{~h}$ ), which suggested that the antiproliferative effect of BMSCs was not due to their differentiation potentials. Similarly, previous studies have reported that cell stemness does not explain the MSC-mediated repair of a number of tissues $(44,45)$.

Macrophages can inhibit the proliferation of $\mathrm{T}$ cells by NO-mediated reduction of STAT5 phosphorylation in T cells (46). Similarly, BMSCs displayed antiproliferative effects on nicotine exposed-T cells in a NO production-dependent manner in the present study. Moreover, in a previous in vivo study, adoptive transfer of BMSCs also led to significantly 
reduced STAT5 phosphorylation in rat lung $\mathrm{T}$ lymphocytes after chronic direct exposure to cigarette smoke. In the present study, the direct BMSC:T cell in vitro co-culture resulted in increased phosphorylated STAT5 and decreased total STAT5 expression in T cells. Additionally, L-NAME reversed the reduction of STAT5 phosphorylation induced by BMSCs, which suggested that NO functionally suppressed STAT5 to inhibit T-cell proliferation, as STAT5 is a crucial transcription factor for $\mathrm{T}$ cell activation and proliferation $(47,48)$. However, neither shRNA-mediated silencing of iNOS or L-NAME treatment completely reversed the BMSC-induced inhibition of T-cell proliferation, suggesting that NO might not be the only factor responsible for the antiproliferative effect of BMSCs on T cells. In the present study, $\sim 80 \%$ of T-cell proliferation was restored after functional inactivation of iNOS, indicating that NO may serve as the major soluble factor responsible for the suppressive function of BMSCs. Consistently, BMSCs derived from mice also inhibit T-cell proliferation via NO production (20), which together with the results of the present study and a previous study (23) indicated that MSCs from mice and rats could be categorized as iNOS-utilizing immunosuppressors (42). Collectively, inhibition of T-cell proliferation by BMSC-derived NO and subsequent inhibition of STAT5 phosphorylation in $\mathrm{T}$ cells may partially explain BMSC-mediated alleviation of chronic inflammation and lung injury in rats exposed to long-term chronic cigarette smoke.

$\mathrm{T}$ cells are classified into $\mathrm{CD} 4^{+}$and $\mathrm{CD} 8^{+} \mathrm{T}$ cells, depending on the surface expression of CD4 and CD8 molecules. A clinical study revealed that a high number of $\mathrm{CD}^{+} \mathrm{T}$ cells infiltrate the airway wall and lung parenchyma of patients with COPD, and the number of $\mathrm{CD}^{+} \mathrm{T}$ cells is positively correlated with the severity of airflow limitation and emphysema (10). Maeno et al (49) reported that cigarette smoke exposure did not induce emphysema-like changes in $\mathrm{CD}^{+} \mathrm{T}$ cell-deficient mice, suggesting that $\mathrm{CD}^{+} \mathrm{T}$ cell-mediated inflammation is involved in the development of COPD and emphysema. However, the precise mechanisms underlying the regulation of T-lymphocyte differentiation by BMSCs during the modulation of chronic lung inflammation and injury in animal models of COPD requires further investigation. A limitation of the present study was that total T cells were used in the co-culture experiments. Both $\mathrm{CD}^{+}$ and $\mathrm{CD}^{+} \mathrm{T}$ cells have been implicated in the disease progression of COPD; therefore, characterizing the $\mathrm{T}$ cell subtype that is more susceptible to BMSC-mediation and $\mathrm{NO}$ is required to improve the knowledge of the suppressive function of BMSCs. In addition, whether $\mathrm{CD} 4^{+}$and $\mathrm{CD}^{+} \mathrm{T}$ cells exhibit differential STAT5 phosphorylation following NO stimulation or co-culture with BMSCs also requires further investigation. Another limitation of the present study was that the T cells were isolated from rat spleens and not from rat lungs due to low abundance; therefore, spleen-derived T cells might not fully represent the phenotype of cigarette smoke-exposed lung-derived T cells.

In summary, an in vitro BMSC:T cell co-culture system was established and the antiproliferative effect of rat BMSCs on splenic $\mathrm{T}$ cells isolated from rats after long-term chronic cigarette smoke exposure was investigated. Functional inactivation of iNOS in BMSCs with shRNA-mediated silencing or specific inhibitor treatment indicated that BMSC-induced inhibition of T-cell proliferation was mediated by iNOS expression and NO-induced reduction of STAT5 phosphorylation in T cells.
The present study provided direct evidence of the suppressive effect of BMSCs on cigarette-exposed T cells and could, at least partially, explain the mechanisms underlying the beneficial roles of BMSC infusion in animal models and patients. Furthermore, the present study indicated that adoptive transfer of BMSCs may serve as a promising therapeutic strategy for chronic smoking-induced COPD and emphysema.

\section{Acknowledgements}

The authors would like to thank Dr Huaping Zhang and Dr Xiaoyan Zhai from the Central Laboratory of Shanxi Medical University for their insightful discussions. The authors would also like to thank Dr Jiahui Zhao from the Central Laboratory of Shanxi Medical University for their technical assistance on western blotting experiments. The authors would like to thank Dr Rui Wang from the Physiology Laboratory of Shanxi Medical University for the critical review and suggestions of the manuscript.

\section{Funding}

The present study was supported by the Ministry of Education (grant no. 2016BY075) and the Health Committee of Shanxi Province in China (grant no. 201601011).

\section{Availability of data and materials}

The datasets used and/or analyzed during the present study are available from the corresponding author on reasonable request.

\section{Authors' contributions}

JX and XL conceived and designed the study. XL and PL designed the methodology. XL and PL performed the experiments and acquired the data. XL analyzed and interpreted the data, wrote the manuscript and provided supervision. All authors read and approved the final manuscript.

\section{Ethics approval and consent to participate}

The present study was approved by the Animal Care and Research Committee of Shanxi Medical University and performed with strict adherence to the Guide for the Care and Use of Laboratory Animals (8th edition).

\section{Patient consent for publication}

Not applicable.

\section{Competing interests}

The authors declare that they have no competing interests.

\section{References}

1. GBD 2015 Disease and Injury Incidence and Prevalence Collaborators: Global, regional, and national incidence, prevalence, and years lived with disability for 310 diseases and injuries, 1990-2015: A systematic analysis for the global burden of disease study 2015. Lancet 388: 1545-1602, 2016. 
2. GBD 2015 Mortality and Causes of Death Collaborators: Global, regional, and national life expectancy, all-cause mortality, and cause-specific mortality for 249 causes of death, 1980-2015: A systematic analysis for the global burden of disease study 2015 . Lancet 388: 1459-1544, 2016.

3. Decramer M, Janssens W and Miravitlles M: Chronic obstructive pulmonary disease. Lancet 379: 1341-1351, 2012.

4. Salvi S: Tobacco smoking and environmental risk factors for chronic obstructive pulmonary disease. Clin Chest Med 35: 17-27, 2014.

5. Strzelak A, Ratajczak A, Adamiec A and Feleszko W: Tobacco smoke induces and alters immune responses in the lung triggering inflammation, allergy, asthma and other lung diseases: A mechanistic review. Int J Environ Res Public Health 15: pii: E1033, 2018.

6. Qiu F, Liang CL, Liu H, Zeng YQ, Hou S, Huang S, Lai X and Dai Z: Impacts of cigarette smoking on immune responsiveness: Up and down or upside down? Oncotarget 8: 268-284, 2017.

7. Gadgil A and Duncan SR: Role of T-lymphocytes and pro-inflammatory mediators in the pathogenesis of chronic obstructive pulmonary disease. Int J Chron Obstruct Pulmon Dis 3: 531-541, 2008.

8. Nurwidya F, Damayanti T and Yunus F: The role of innate and adaptive immune cells in the immunopathogenesis of chronic obstructive pulmonary disease. Tuberc Respir Dis (Seoul) 79 : $5-13,2016$

9. Rinaldi M, Maes K, De Vleeschauwer S, Thomas D, Verbeken EK, Decramer M, Janssens W and Gayan-Ramirez GN: Long-term nose-only cigarette smoke exposure induces emphysema and mild skeletal muscle dysfunction in mice. Dis Model Mech 5: 333-341, 2012

10. Sullivan AK, Simonian PL, Falta MT, Mitchell JD, Cosgrove GP Brown KK, Kotzin BL, Voelkel NF and Fontenot AP: Oligoclonal CD4+ T cells in the lungs of patients with severe emphysema. Am J Respir Crit Care Med 172: 590-596, 2005.

11. Hogg JC, Chu F, Utokaparch S, Woods R, Elliott WM, Buzatu L, Cherniack RM, Rogers RM, Sciurba FC, Coxson HO and Paré PD: The nature of small-airway obstruction in chronic obstructive pulmonary disease. N Engl J Med 350: 2645-2653, 2004.

12. D'Hulst AI, Vermaelen KY, Brusselle GG, Joos GF and Pauwels RA: Time course of cigarette smoke-induced pulmonary inflammation in mice. Eur Respir J 26: 204-213, 2005.

13. D'Hulst AI, Maes T, Bracke KR, Demedts IK, Tournoy KG, Joos GF and Brusselle GG: Cigarette smoke-induced pulmonary emphysema in scid-mice. Is the acquired immune system required? Respir Res 6: 147, 2005.

14. Jiang XX, Zhang Y, Liu B, Zhang SX, Wu Y, Yu XD and Mao N: Human mesenchymal stem cells inhibit differentiation and function of monocyte-derived dendritic cells. Blood 105: 4120-4126, 2005.

15. Corcione A, Benvenuto F, Ferretti E, Giunti D, Cappiello V, Cazzanti F, Risso M, Gualandi F, Mancardi GL, Pistoia V and Uccelli A: Human mesenchymal stem cells modulate B-cell functions. Blood 107: 367-372, 2006.

16. Di Nicola M, Carlo-Stella C, Magni M, Milanesi M, Longoni PD, Matteucci P, Grisanti S and Gianni AM: Human bone marrow stromal cells suppress T-lymphocyte proliferation induced by cellular or nonspecific mitogenic stimuli. Blood 99: 3838-3843, 2002

17. Krampera M, Glennie S, Dyson J, Scott D, Laylor R, Simpson E and Dazzi F: Bone marrow mesenchymal stem cells inhibit the response of naive and memory antigen-specific $\mathrm{T}$ cells to their cognate peptide. Blood 101: 3722-3729, 2003

18. Tse WT, Pendleton JD, Beyer WM, Egalka MC and Guinan EC Suppression of allogeneic T-cell proliferation by human marrow stromal cells: Implications in transplantation. Transplantation 75 389-397, 2003.

19. Rasmusson I, Ringden O, Sundberg B and Le Blanc K: Mesenchymal stem cells inhibit lymphocyte proliferation by mitogens and alloantigens by different mechanisms. Exp Cell Res 305: 33-41, 2005.

20. Sato K, Ozaki K, Oh I, Meguro A, Hatanaka K, Nagai T, Muroi K and Ozawa K: Nitric oxide plays a critical role in suppression of T-cell proliferation by mesenchymal stem cells. Blood 109 228-234, 2007

21. Wang M, Yuan Q and Xie L: Mesenchymal stem cell-based immunomodulation: Properties and clinical application. Stem Cells Int 2018: 3057624, 2018.
22. Yagi H, Soto-Gutierrez A, Parekkadan B, Kitagawa Y, Tompkins RG, Kobayashi N and Yarmush ML: Mesenchymal stem cells: Mechanisms of immunomodulation and homing. Cell Transplant 19: 667-679, 2010.

23. Zinöcker S and Vaage JT: Rat mesenchymal stromal cells inhibit $\mathrm{T}$ cell proliferation but not cytokine production through inducible nitric oxide synthase. Front Immunol 3: 62, 2012.

24. Li X, Wang J, Cao J, Ma L and Xu J: Immunoregulation of bone marrow-derived mesenchymal stem cells on the chronic cigarette smoking-induced lung inflammation in rats. Biomed Res Int 2015: 932923, 2015.

25. Vig M, Srivastava S, Kandpal U, Sade H, Lewis V, Sarin A, George A, Bal V, Durdik JM and Rath S: Inducible nitric oxide synthase in $\mathrm{T}$ cells regulates $\mathrm{T}$ cell death and immune memory. J Clin Invest 113: 1734-1742, 2004.

26. National Research Council (US) Committee for the Update of the Guide for the Care and Use of Laboratory Animals. Guide for the Care and Use of Laboratory Animals. 8th edition. Washington (DC), National Academies Press (US), 2011.

27. Mendicino M, Bailey AM, Wonnacott K, Puri RK and Bauer SR: MSC-based product characterization for clinical trials: An FDA perspective. Cell Stem Cell 14: 141-145, 2014.

28. Wulfing C, Sjaastad MD and Davis MM: Visualizing the dynamics of $\mathrm{T}$ cell activation: Intracellular adhesion molecule 1 migrates rapidly to the $\mathrm{T}$ cell/B cell interface and acts to sustain calcium levels. Proc Natl Acad Sci USA 95: 6302-6307, 1998.

29. Livak KJ and Schmittgen TD: Analysis of relative gene expression data using real-time quantitative PCR and the 2(-Delta Delta C(T)) method. Methods 25: 402-408, 2001

30. Barnes PJ and Cosio MG: Characterization of T lymphocytes in chronic obstructive pulmonary disease. PLoS Med 1: e20, 2004.

31. Ni L and Dong C: Roles of myeloid and lymphoid cells in the pathogenesis of chronic obstructive pulmonary disease. Front Immunol 9: 1431, 2018.

32. Antunes MA, Lapa E Silva JR and Rocco PR: Mesenchymal stromal cell therapy in COPD: From bench to bedside. Int J Chron Obstruct Pulmon Dis 12: 3017-3027, 2017.

33. Broekman W, Khedoe P, Schepers K, Roelofs H, Stolk J and Hiemstra PS: Mesenchymal stromal cells: A novel therapy for the treatment of chronic obstructive pulmonary disease? Thorax 73 : $565-574,2018$

34. Kruk DMLW, Heijink IH, Slebos DJ, Timens W and Ten Hacken NH: Mesenchymal stromal cells to regenerate emphysema: On the horizon? Respiration 96: 148-158, 2018.

35. Maitra B, Szekely E, Gjini K, Laughlin MJ, Dennis J, Haynesworth SE and Koç ON: Human mesenchymal stem cells support unrelated donor hematopoietic stem cells and suppress T-cell activation. Bone Marrow Transplant 33: 597-604, 2004.

36. Beyth S, Borovsky Z, Mevorach D, Liebergall M, Gazit Z, Aslan H, Galun E and Rachmilewitz J: Human mesenchymal stem cells alter antigen-presenting cell maturation and induce T-cell unresponsiveness. Blood 105: 2214-2219, 2005.

37. Groh ME, Maitra B, Szekely E and Koç ON: Human mesenchymal stem cells require monocyte-mediated activation to suppress alloreactive T cells. Exp Hematol 33: 928-934, 2005.

38. Meisel R, Zibert A, Laryea M, Gobel U, Daubener W and Dilloo D: Human bone marrow stromal cells inhibit allogeneic T-cell responses by indoleamine 2,3-dioxygenase-mediated tryptophan degradation. Blood 103: 4619-4621, 2004

39. Glennie S, Soeiro I, Dyson PJ, Lam EW and Dazzi F: Bone marrow mesenchymal stem cells induce division arrest anergy of activated T cells. Blood 105: 2821-2827, 2005.

40. Djouad F, Plence P, Bony C, Tropel P, Apparailly F, Sany J, Noel D and Jorgensen C: Immunosuppressive effect of mesenchymal stem cells favors tumor growth in allogeneic animals. Blood 102: 3837-3844, 2003.

41. Laing AG, Fanelli G, Ramirez-Valdez A, Lechler RI, Lombardi G and Sharpe PT: Mesenchymal stem cells inhibit T-cell function through conserved induction of cellular stress. PLoS One 14: e0213170, 2019.

42. Su J, Chen X, Huang Y, Li W, Li J, Cao K, Cao G, Zhang L, Li F, Roberts AI, et al: Phylogenetic distinction of iNOS and IDO function in mesenchymal stem cell-mediated immunosuppression in mammalian species. Cell Death Differ 21: 388-396, 2014.

43. Shi Y, Su J, Roberts AI, Shou P, Rabson AB and Ren G: How mesenchymal stem cells interact with tissue immune responses. Trends Immunol 33: 136-143, 2012.

44. Prockop DJ: 'Stemness' does not explain the repair of many tissues by mesenchymal stem/multipotent stromal cells (MSCs). Clin Pharmacol Ther 82: 241-243, 2007. 
45. Stagg J: Immune regulation by mesenchymal stem cells: Two sides to the coin. Tissue Antigens 69: 1-9, 2007.

46. Mazzoni A, Bronte V, Visintin A, Spitzer JH, Apolloni E, Serafini P, Zanovello P and Segal DM: Myeloid suppressor lines inhibit $\mathrm{T}$ cell responses by an NO-dependent mechanism. J Immunol 168: 689-695, 2002.

47. Owen DL and Farrar MA: STAT5 and CD $4{ }^{+} \mathrm{T}$ cell immunity. F1000Res 6: 32, 2017.
48. Tripathi P, Kurtulus S, Wojciechowski S, Sholl A, Hoebe K, Morris SC, Finkelman FD, Grimes HL and Hildeman DA: STAT5 is critical to maintain effector CD8+ T cell responses. J Immunol 185: 2116-2124, 2010.

49. Maeno T, Houghton AM, Quintero PA, Grumelli S, Owen CA and Shapiro SD: CD8+ T cells are required for inflammation and destruction in cigarette smoke-induced emphysema in mice. J Immunol 178: 8090-8096, 2007. 